\title{
Non-Quasi-Static Large-Signal Model for RF LDMOS Power Transistors
}

\begin{abstract}
In this paper, we propose a non-quasi-static large-signal model to capture the high-frequency dispersion exhibited by laterally diffused metal-oxide semiconductor (LDMOS) devices. We show that industry-standard nonlinear large-signal models for LDMOS based on quasi-static assumptions are not sufficient for high-efficiency designs at frequencies higher than 2 GHz. This dispersive behavior results from the combination of high-frequency operation and the lengthened drain extension region that is needed to support high-voltage operation. To improve the model accuracy, higher-order current and charge components, which are directly integrated from bias-dependent $S$-parameter data, are included in the model. The non-quasi-static large-signal model improves the efficiency and gain predictions by $10 \%$ and $0.5 \mathrm{~dB}$ at 3.5 GHz. These improvements in accuracy are essential for power amplifier designers to achieve the performance targets necessary for $4 \mathrm{G}$ and upcoming $5 \mathrm{G}$ designs.
\end{abstract}

Index Terms-Frequency dispersion, LDMOS, non-quasistatic, transistor modeling.

\section{INTRODUCTION}

The silicon laterally diffused metal-oxide semiconductor (LDMOS) devices have been the main semiconductor technology for base station power amplifiers over the last several decades. As wireless communication systems continue to evolve, the requirement for more spectrum at higher frequencies increases the demands on radio frequency (RF) device technologies. Recent research has shown that LDMOS power transistors exhibit high-frequency dispersion resulting from their device construction [1]-[3]. As the wireless market drives increased operating frequency past $3 \mathrm{GHz}$, the need for accurate LDMOS transistor models is essential.

The high-frequency dispersion can be captured by non-quasistatic (NQS) models. An equivalent circuit view of the nonquasi-static small-signal model is shown in Fig. 1(a). Its quasistatic (QS) counterpart, which omits the $\mathrm{R}_{\mathrm{gg}}$, $\mathrm{R}_{\mathrm{gd}}$, and the time delay $\tau$ in the small-signal gain term, is often used for modeling simplification. Without the RC time constants and the resulting delay time, the simplified quasi-static model can lead to inaccurate results when the time constants are comparable to the inverse of excitation frequency. The majority of nonlinear electro-thermal models used to describe LDMOS transistors are based on quasi-static assumptions, and there have been previous research attempts to incorporate non-quasi-static effects in small-signal modeling [2] and large-signal modeling for 2.1 $\mathrm{GHz}$ application [3]. These modeling methods use additional nonlinear resistors and capacitors to represent the time delay and time constants, and complex model extraction procedure is

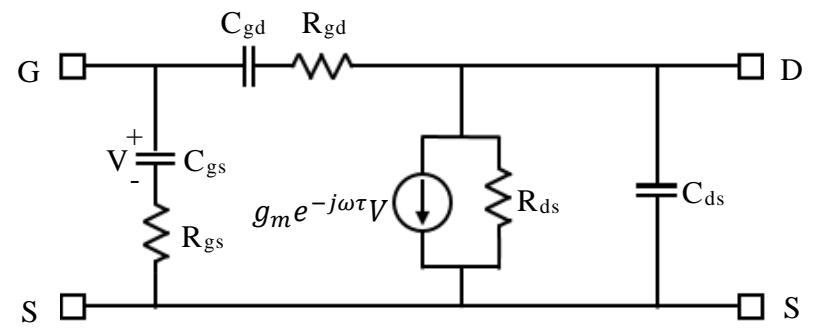

(a)

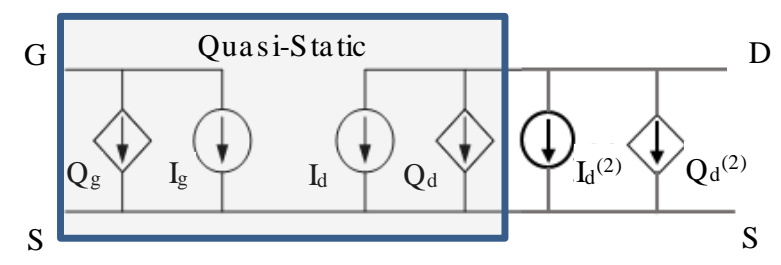

(b)

Fig. 1. Circuit representation of (a) the small-signal non-quasi-static model and (b) the proposed large-signal non-quasi-static model, composed of the quasi-static approximation and higher-order current and charge sources.

usually required to obtain these component values. In [4], a simplified complex gain term was used, where the transconductance $g_{m}$ and its associated time delay $\tau$ are represented by the first order Taylor series:

$$
\mathrm{g}_{\mathrm{m}} e^{-j \omega \tau} \approx \mathrm{g}_{\mathrm{m}}-j \omega \mathrm{g}_{\mathrm{m}} \tau
$$

Such simplification is valid when the operating frequency is relatively low, under which the higher order terms of Taylor series are negligible. However, this may not be sufficient for accurate modeling under high-frequency operation. Recent work in [1] shows the high-frequency dispersion in LDMOS devices as a consequence of the high-voltage operation and the length of the drain extension, which is the time needed by the electrons to travel from the drain to the channel beneath the gate (also known as drain extension or drift region). To model this transit time and capture high-frequency dispersion, a rigorous nonquasi-static model becomes necessary for operation in the 3-5 $\mathrm{GHz}$ frequency range.

In this paper, we present a new non-quasi-static large-signal model for LDMOS devices, with higher-order current and charge sources. This work is based on [5] and is the first time to be applied to LDMOS device modeling. These higher-order current and charge sources are used to compensate the nonquasi-static effect, and are directly extracted from the pulsed S- 
parameter measurement data at multiple biases, through an automated numerical integration procedure. The model has a simple topology and can be conveniently implemented in any commercial circuit simulators. It can also be used to augment existing models by adding parallel branches of higher-order current and charge sources. The paper is organized as follows. The frequency dispersion within LDMOS transistors will be presented through modeling the small-signal behavior across a wide frequency range, based on which the large-signal non-quasi-static formulation will be derived. A table-based non-quasi-static model will be proposed, and its implementation in commercial circuit simulators will be discussed. Validation of the tablebased NQS model at $3.5 \mathrm{GHz}$ with accuracy improvement over a conventional quasi-static model will be demonstrated.

\section{High-FREQUENCY DiSPERSION EFFECT IN LDMOS}

\section{A. Quasi-Static Assumption}

Under the quasi-static assumption, the first-order Taylor series expansion in (1) is often used. The large-signal nonlinear model with quasi-static assumption can then be represented by a simplified topology with nonlinear current and charge sources between gate-to-source and drain-to-source terminals [4], shown in Fig. 1(b). The $g_{m}$ term in (1) contributes to the nonlinear drain-to-source current component $I_{d}$, and the $-j \omega g_{m} \tau$ term is converted into a trans-capacitance and contributes to the nonlinear drain-to-source charge component $Q_{d}$.

To illustrate the need for higher-order components, a smallsignal model was extracted from measured $S$-parameters of a 5 $\mathrm{mm}$ 10-finger 28-V RF LDMOS transistor, biased at $6 \mathrm{~mA} / \mathrm{mm}$ (class $\mathrm{AB}$ ) and $28 \mathrm{~V}$ drain voltage. In the small-signal model, various orders $(1,2$, and 3$)$ of the Taylor series for the complex gain term was used. As shown in Fig. 2, the S-parameters were only accurate up to $2 \mathrm{GHz}$ for the model with the 1 st order Taylor approximation. In other words, the model with the first-order Taylor approximation in [4] can only match the actual transconductance behavior below $2 \mathrm{GHz}$. To accurately represent the transconductance behavior in a broad band up to $10 \mathrm{GHz}$, at least 3rd order Taylor series are needed. This shows the necessity of a non-quasi-static model with higher-order components for high-frequency dispersion modeling.
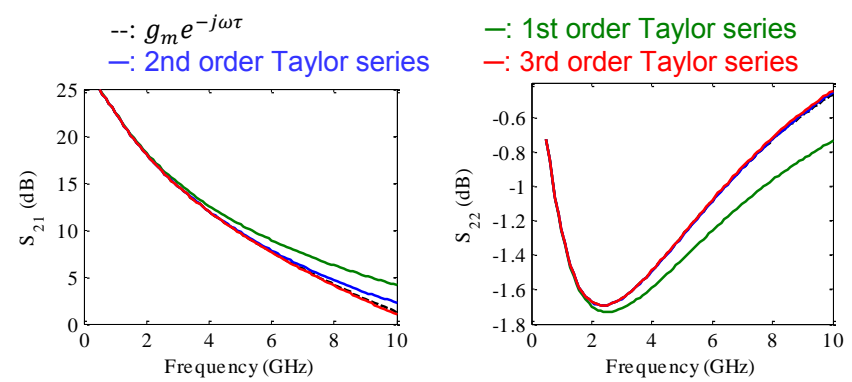

Fig. 2. Inclusion of the additional terms in the Taylor series expansion improves the fit to S-parameters at higher frequencies. Plotted above are multiple orders of the expansion showing improved accuracy with more terms.

\section{B. Non-Quasi-Static Model Formulation}

The complete non-quasi-static formulation with higher-order currents and charges were first presented in [5]. In this paper, we propose to use a simplified version for LDMOS modeling. From the observation in Section II.A, the higher-order Taylor expansion terms are important in matching up the high-frequency S-parameters. The third-order Taylor series of the transconductance can be written as:

$$
\mathrm{g}_{\mathrm{m}} e^{-j \omega \tau} \approx \mathrm{g}_{\mathrm{m}}\left(1-j \omega \tau+\frac{(-j \omega \tau)^{2}}{2}+\frac{(-j \omega \tau)^{3}}{6}\right)
$$

The large-signal non-quasi-static drain current model can be formulated in addition to the existing quasi-static model as [5][6]:

$$
\begin{aligned}
I_{\mathrm{d}}^{(0)} & =\int_{V_{g s 0}}^{V_{g s}} g_{m}\left(v_{g s}, V_{d s 0}\right) d v_{g s}+\int_{V_{d s 0}}^{V_{d s}} g_{d s}\left(V_{g s}, v_{d s}\right) d v_{d s} \\
& +I_{d}\left(V_{g s 0}, V_{d s 0}\right) \\
Q_{\mathrm{d}}^{(0)} & =\int_{V_{g s 0}}^{V_{g s}}\left[C_{m}\left(v_{g s}, V_{d s 0}\right)-C_{g d}\left(v_{g s}, V_{d s 0}\right)\right] d v_{g s} \\
& +\int_{V_{d s 0}}^{V_{d s}}\left[C_{d s}\left(V_{g s}, v_{d s}\right)+C_{g d}\left(V_{g s}, v_{d s}\right)\right] d v_{d s} \\
& +Q_{d}\left(V_{g s 0}, V_{d s 0}\right) \\
I_{\mathrm{d}}^{(2)} & =\int_{V_{g s 0}}^{V_{g s}}\left[g_{m}\left(v_{g s}, V_{d s 0}\right) \cdot \frac{1}{2} \tau^{2}\left(v_{g s}, V_{d s 0}\right)\right] d v_{g s} \\
Q_{\mathrm{d}}^{(2)} & =\int_{V_{g s 0}}^{V_{g s}}\left[-g_{m}\left(v_{g s}, V_{d s 0}\right) \cdot \frac{1}{6} \tau^{3}\left(v_{g s}, V_{d s 0}\right)\right] d v_{g s}
\end{aligned}
$$

Equations (3) and (4) are derived with the quasi-static assumption, and are sufficient to model devices for low-frequency or narrow-band modeling. This type of models has been well adopted and has shown very good behavior when the frequency-dispersion is not present [6]. Equations (5) and (6) are derived to represent the second and third order of the Taylor approximation. As shown in Fig. 1(b), they are implemented as second order current and charge sources, and can be extracted directly from the small-signal S-parameter measurements through numerical integration. The implementation of the higher-order current and charge sources is also straightforward and a data table can be conveniently used in any commercial circuit simulator. Due to the simple topology, these higher-order sources can be added to any quasi-static model for accuracy enhancement. 


\section{EXAMPLE}

The proposed non-quasi-static formulation is applied to model the $5 \mathrm{~mm}$ 10-finger 28-V RF LDMOS transistor referred earlier in Section II, where an existing quasi-static model is available and shows reasonable accuracy for a $2 \mathrm{GHz}$ design. However, when the quasi-static model is used to design at 3.5 $\mathrm{GHz}$, there is a significant over-prediction in efficiency and power. From Fig. 2, we expect to see this behavior since the frequency dispersion at $3.5 \mathrm{GHz}$ is not neglectable. In addition, the nonlinear model needs to be accurate across a wide frequency range usually up to the third harmonics of the operating band, where the higher-order components in (5) and (6) are extremely important to achieve such accuracy improvement.

A large-signal quasi-static model is created based on the existing quasi-static model, adding the second order current and charge sources as shown in Fig. 1(b), in a data table format. Fig. 3 shows the comparison of high-frequency current between measurement data, the proposed non-quasi-static model, and the quasi-static model. The high-frequency currents are compared at multiple frequencies, as well as against the measured DC current. It is shown that as the frequency increases, the high-frequency current of the quasi-static model remains close to the DC current but deviates away from the actual device behavior. The non-quasi-static model can compensate the differences and matches well with the measurement data.

Large-signal load pull simulations were also performed for the two models, under class $\mathrm{AB}$ bias and operating frequency at $3.5 \mathrm{GHz}$. Table I compares the output power and efficiency at a load impedance matched for best efficiency. The drive-up plots for output power and drain efficiency are also compared

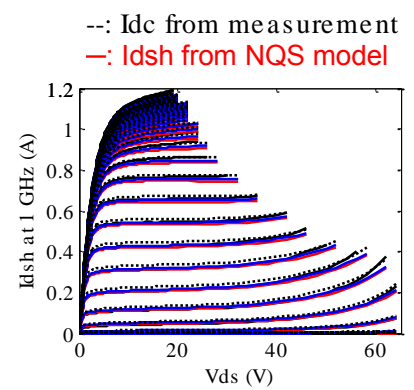

(a)

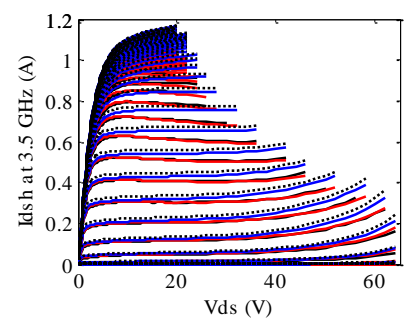

(c)

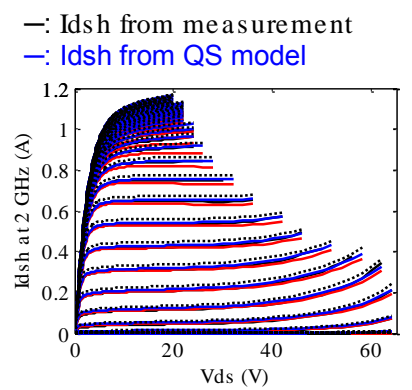

(b)

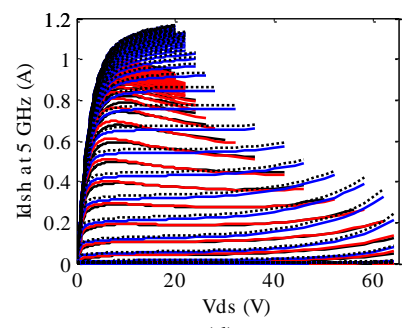

(d)
Fig. 3. Comparison of the high-frequency current Idsh at (a) $1 \mathrm{GHz}$, (b) 2 $\mathrm{GHz}$, (c) $3.5 \mathrm{GHz}$, and (d) $5 \mathrm{GHz}$, between the Idsh extracted from measurement data, the proposed non-quasi-static model, the quasi-satic model, and the measured DC current Idc. The high-frequency currents were obtained through numerical integration as presented in [1]. It is clearly shown that as frequency increases, the NQS model is needed to correct the overprediction in high-frequency current from the QS model only.

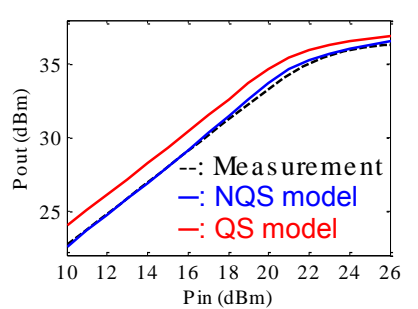

(a)

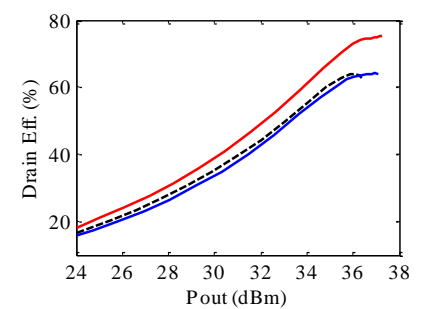

(b)
Fig. 4. Comparison of (a) output power and (b) efficiency between load pull measurement data, the NQS model, and the QS model. The device and models were biased at Idq of $30 \mathrm{~mA}$ and $\mathrm{Vds}$ of $28 \mathrm{~V}$, under $3.5 \mathrm{GHz}$ operating frequency with load matched for maximum efficiency.

\section{TABLE I}

LARGE-SignAl LOAD PUll PERFORMANCE AT 3.5 GHz, 3dB GAIN COMPRESSION, BIASED AT $6 \mathrm{~mA} / \mathrm{mm}$ AND LOAD MATCHED FOR BEST EFFICIENCY

\begin{tabular}{|c|c|c|c|c|}
\hline & $\begin{array}{c}\text { Idq } \\
(\mathbf{m A})\end{array}$ & $\begin{array}{c}\text { Pout } \\
(\mathbf{d B m})\end{array}$ & $\begin{array}{c}\text { Gt } \\
(\mathbf{d B})\end{array}$ & $\begin{array}{c}\text { Drain Eff. } \\
(\boldsymbol{\%})\end{array}$ \\
\hline Mea surement & 25.00 & 36.67 & 10.28 & 63.52 \\
\hline QS Model & 25.04 & 37.06 & 11.28 & 74.39 \\
\hline NQS Mode1 & 24.98 & 36.55 & 10.69 & 63.85 \\
\hline
\end{tabular}

in Fig. 4. Compared to the quasi-static model, the non-quasistatic model predicts power and efficiency much closer to the measured performance.

\section{CONCLUSION}

In this paper, a new non-quasi-static large-signal model was presented to characterize the high-frequency dispersion behavior in LDMOS transistors. Higher-order current and charge sources were added to an existing quasi-static model to improve its performance under high frequency operation. The proposed non-quasi-static model was capable of predicting the high-frequency operation behavior accurately.

\section{REFERENCES}

[1] P. H. Aaen, L. Zhang, and K. Kim, "Characterization and modeling of frequency dispersion in RF LDMOS transistors," in 11th European Microwave Integrated Circuits Conference (EuMIC), London, UK, Oct. 2016, pp. 49-52.

[2] P. Roblin, S. Akhtar, and J. Strahler, "New non-quasi-static theory for extracting small-signal parameters applied to ldmosfets," IEEE Microwave and Guided Wave Letters, vol. 10, no. 8, pp. 322-324, Aug. 2000.

[3] R. Follmann, et al., "A new precise large signal LDMOS(T) model including time delay effects," in 2004 IEEE MTT-S Int. Microwave Symposium Digest, Fort Worth, TX, Oct. 2004, pp. 1061-1064.

[4] P. H. Aaen, J. Pla, and J. Wood, Modeling and Characterization of RF and Microwave Power FETs. Cambridge University Press, 2007.

[5] S. Homayouni, D. Schreurs, G. Crupi, and B. Nauwelaers, "Technology independent non-quasi-static table-based nonlinear model generation," IEEE Trans. Microw. Theory Tech., vol. 57, no. 12, pp. 2845-2852, Dec. 2009.

[6] J. Wood, et al., "A nonlinear electro-thermal scalable model for highpower RF LDMOS transistors," IEEE Trans. Microw. Theory Tech., vol. 57, no. 2, pp. 282-292, Feb. 2009. 